October 22, 2018

\title{
Production of Two Pions Induced by Neutrinos
}

\author{
M. Valverde and J. Nieves \\ Departamento de Física Atómica, Molecular y Nuclear, \\ Universidad de Granada, E-18071 Granada, Spain. \\ E. Hernández \\ Grupo de Física Nuclear, Departamento de Física Fundamental e IUFFyM, \\ Universidad de Salamanca, E-37008 Salamanca, Spain. \\ S.K. Singh \\ Department of Physics, Aligarh Muslim University, Aligarh-202002, India. \\ M.J. Vicente Vacas \\ Departamento de Física Teórica and IFIC, Centro Mixto Universidad de Valencia-CSIC \\ Institutos de Investigación de Paterna, Aptdo. 22085, E-46071 Valencia, Spain.
}

\begin{abstract}
We study the threshold production of two pions induced by neutrinos in nucleon targets. The contribution of nucleon pole, pion and contact terms is calculated using a chiral Lagrangian. The contribution of the Roper resonance, neglected in earlier studies, has also been taken into account.
\end{abstract}

\section{INTRODUCTION}

A proper and precise understanding of the processes induced by neutrino interactions is required in the analysis of neutrino oscillation experiments. For instance, at intermediate energies, above $0.5 \mathrm{GeV}$, one pion production becomes relevant. Most of the theoretical models for this reaction assume the dominance of $\Delta(1232)$ resonance mechanism [1, 2, 3, 4], but others also include background terms [5, 6, 7, 8]. Above these energies new baryonic resonances can be excited, the first of these resonances being the Roper $N^{*}(1440)$ which has a sizable decay into a scalar pion pair and it is very wide. However, the $\Delta$ does not couple to two pions in $s$-wave and thus it is not relevant at energies where only slow pions are produced.

There exist very few attempts to measure the two pion production induced by neutrinos and antineutrinos. Experiments done at ANL [9, 10] and BNL[11] investigated the two pion production processes, in order to test the predictions of chiral symmetry. Biswas et al. [12] used PCAC and current algebra methods to calculate the threshold production of two pions. Adjei et al. [13] made specific predictions using an effective Lagrangian incorporating chiral symmetry. However, these models did not include any resonance production, as we do. Furthermore we use an expansion of the chiral Lagrangian that includes terms up to $\mathcal{O}\left(1 / f_{\pi}^{3}\right)$, while Adjei et al. kept only terms up to $\mathcal{O}\left(1 / f_{\pi}^{2}\right)$. More detailed discussions can be found in Ref. [14].

\section{PION PRODUCTION MODEL}

We will focus on the neutrino-pion production reaction off the nucleon driven by charged currents,

$$
\nu_{l}(k)+N(p) \rightarrow l^{-}\left(k^{\prime}\right)+N\left(p^{\prime}\right)+\pi\left(k_{\pi_{1}}\right)+\pi\left(k_{\pi_{2}}\right) .
$$

For the derivation of the hadronic current we use the effective Lagrangian given by the $\mathrm{SU}(2)$ non-linear $\sigma$ model. This model [6] provides us with expressions for the non-resonant hadronic currents that couple with the lepton current, in terms of the first sixteen Feynman diagrams depicted in Fig. 1 .

We also include the two mechanisms depicted in the bottom of Fig 1, which account for the Roper production and its decay into two pions in a $s$-wave isoscalar state. The coupling of the Roper to the charged weak current is written in terms of the current

$$
J_{c c *}^{\alpha}=\frac{F_{1}^{V *}\left(q^{2}\right)}{\mu^{2}}\left(q^{\alpha} \not q-q^{2} \gamma^{\alpha}\right)+i \frac{F_{2}^{V *}\left(q^{2}\right)}{\mu} \sigma^{\alpha \nu} q_{\nu}-G_{A} \gamma^{\alpha} \gamma_{5}-\frac{G_{P}}{\mu} q^{\alpha} \phi \gamma_{5}-\frac{G_{T}}{\mu} \sigma^{\alpha \nu} q_{\nu} \gamma_{5},
$$

which is the most general form compatible with conservation of the vector current. The $G_{T}$ term does not need to vanish; however, most analyses neglect its contribution and we shall do so here. The form factors $G_{A}$ and $G_{P}$ are 

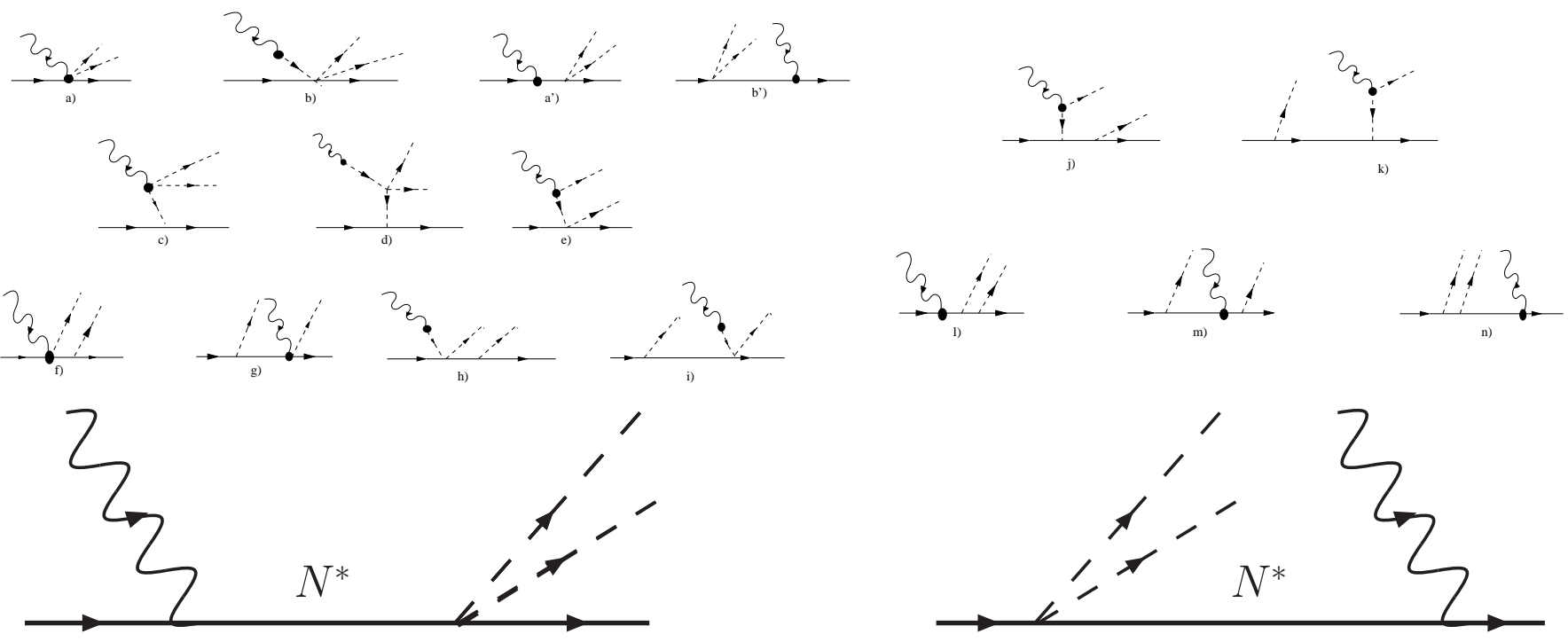

FIG. 1: Top: Nucleon pole, pion pole and contact terms contributing to $2 \pi$ production.Bottom: Direct and crossed Roper excitation contributions to $2 \pi$ production.

constrained by PCAC and the pion pole dominance assumption. The vector form factors $F_{1}^{V *}$ and $F_{2}^{V *}$ can be related to the isovector part of the electromagnetic (EM) form factors. We have fitted the proton-Roper EM transition form factors [15] to the experimental results for helicity amplitudes [16, 17], using a modiffied dipole parametrization (labeled FF1). The Roper EM data have large error bars and it is possible to accommodate quite different functional forms and values for these FF. Thus, we shall consider other different models for the vector form factors: the constituent quark model of Meyer et al. [18] (FF2), the parametrizations of Lalakulich et al. 19] (FF3) and finally the predictions of the recent MAID 20] analysis (FF4).

\section{RESULTS}

In Fig. 2, we present results for the cross section for the process $\nu n \rightarrow \mu^{-} p \pi^{+} \pi^{-}$. We show separately the contribution of the background terms as well as the contribution of the Roper resonance as calculated by using the various form factors described above. The interference between background and the Roper contribution is not shown. We see that the background terms dominate the cross section for neutrino energies $E_{\nu}>0.7 \mathrm{GeV}$. At lower energies the contribution from the Roper could be larger or smaller than the background depending upon the vector form factors used for the $W^{+} N N^{*}$ transition. The differences in the predictions for the cross sections using the various parametrizations could reach a factor two. The Roper contribution is specially sensitive to $F_{2}^{V *}\left(q^{2}\right)$ which is negative in contrast to the positive value which one gets in the case of the nucleon.

We present the results for the cross section for the $\nu n \rightarrow \mu^{-} p \pi^{+} \pi^{-}$channel in left panel of Fig. 3 and for the channel $\nu p \rightarrow \mu^{-} n \pi^{+} \pi^{+}$in the right hand panel. The phase space for these results was restricted following a suggestion by Adjei et al. 13]. We show our results for the first channel with only background terms and with the full model evaluated using the set FF1 of nucleon-Roper transition form factors. Other sets give a similar result in this case. Even in this kinematic region, the theoretical results including the resonance contribution are lower than the experiment. For the second channel there are no contributions from the $N^{*}(1440)$ resonance.

[1] L. Alvarez-Ruso, S. K. Singh and M. J. Vicente Vacas, Phys. Rev. C 57, 2693 (1998).

[2] L. Alvarez-Ruso, S. K. Singh and M. J. Vicente Vacas, Phys. Rev. C 59, 3386 (1999).

[3] E. A. Paschos, J. Y. Yu and M. Sakuda, Phys. Rev. D 69, 014013 (2004).

[4] O. Lalakulich and E. A. Paschos, Phys. Rev. D 71, 074003 (2005).

[5] T. Sato, D. Uno and T. S. H. Lee, Phys. Rev. C 67, 065201 (2003). 


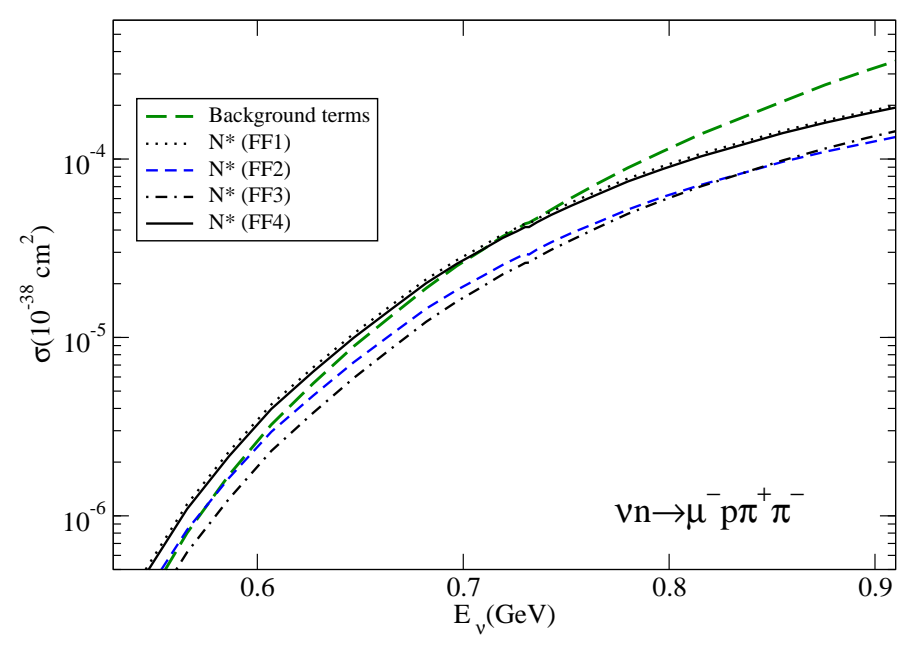

FIG. 2: Cross section for the $\nu n \rightarrow \mu^{-} p \pi^{+} \pi^{-}$reaction as a function of the neutrino energy.
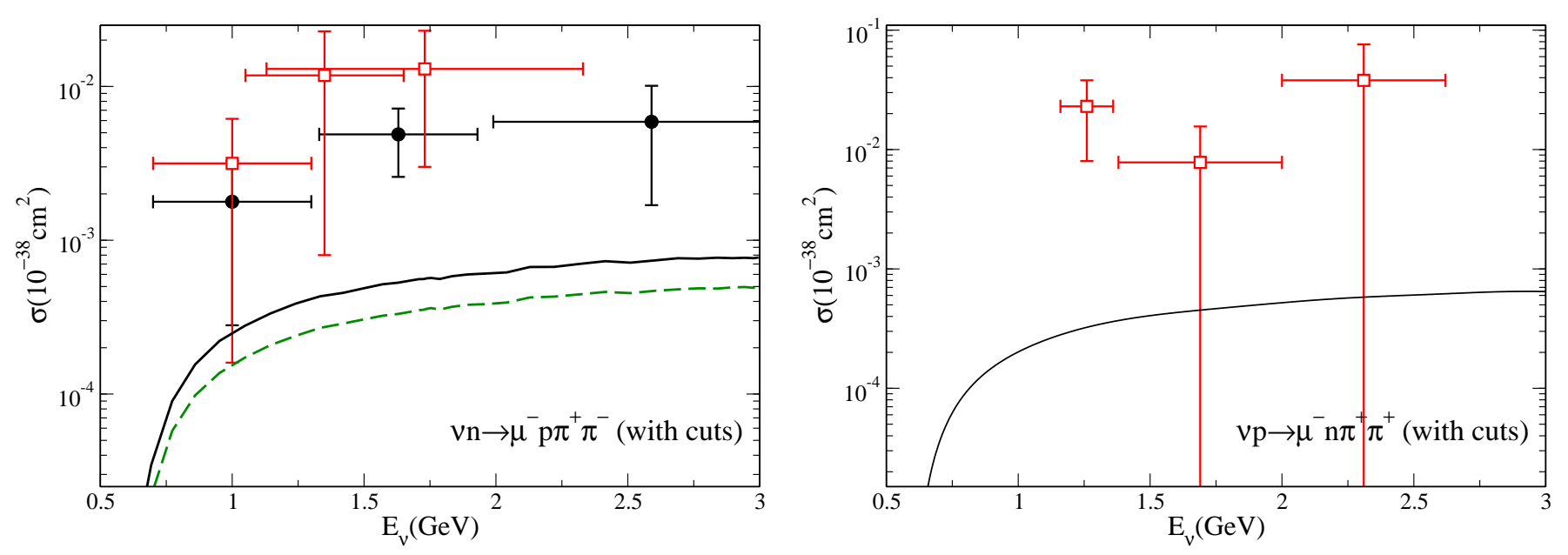

FIG. 3: Cross section for the $\nu n \rightarrow \mu^{-} p \pi^{+} \pi^{-}$(left) and $\nu p \rightarrow \mu^{-} n \pi^{+} \pi^{+}$(rights) with cuts as explained in the text. Dashed line: Background terms. Solid line: Full model with set FF1 of nucleon-Roper transition form factors. Data from Ref. [1] (solid circles) and Ref. [10] (open squares).

[6] E. Hernandez, J. Nieves and M. Valverde, Phys. Rev. D 76, 033005 (2007).

[7] E. Hernandez, J. Nieves and M. Valverde, AIP Conf. Proc. 967, 197 (2007).

[8] E. Hernandez, J. Nieves and M. Valverde, arXiv:0710.5587 [].

[9] S. J. Barish et al. Phys. Rev. D 19, 2521 (1979).

[10] D. Day et al. Phys. Rev. D 28, 2714 (1983).

[11] T. Kitagaki et al. Phys. Rev. D 34, 2554 (1986).

[12] S. N. Biswas, S. R. Choudhury, A. K. Goyal and J. N. Passi, Phys. Rev. D 18, 3187 (1978).

[13] S. A. Adjei, D. A. Dicus and V. L. Teplitz, Phys. Rev. D 23, 672 (1981).

[14] E. Hernandez, J. Nieves, S. K. Singh, M. Valverde and M. J. Vicente Vacas, Phys. Rev. D, in press. arXiv:0710.3562 [].

[15] L. Alvarez-Ruso, M. B. Barbaro, T. W. Donnelly and A. Molinari, Nucl. Phys. A 724, 157 (2003).

[16] I. G. Aznauryan et al. Phys. Rev. C 71, 015201 (2005).

[17] L. Tiator et al. Eur. Phys. J. A 19, 55 (2004).

[18] U. Meyer, E. Hernandez and A. J. Buchmann, Phys. Rev. C 64, 035203 (2001).

[19] O. Lalakulich, E. A. Paschos and G. Piranishvili, Phys. Rev. D 74, 014009 (2006).

[20] D. Drechsel, S. S. Kamalov and L. Tiator, Eur. Phys. J. A 34, 69 (2007). 